\title{
Photo-induced thermal radiation within multilayer optics
}

\author{
Paul Rouquette ${ }^{1,2}$, Claude Amra ${ }^{1}$, Myriam Zerrad ${ }^{1}$, Catherine Grèzes-Besset ${ }^{2}$ \\ ${ }^{1}$ Aix Marseille Univ, CNRS, Centrale Marseille, Institut Fresnel, Marseille France \\ ${ }^{2}$ CILAS, Aubagne, France
}

\begin{abstract}
Exact models of photo-induced temperature and thermal radiation are presented for optical coatings submitted to spatiotemporal regimes. Results are useful to analyze and predict thermal processes in optical thin films at different temporal and spatial scales. They also open a door to control the emissivity patterns of multilayers.
\end{abstract}

Keywords: Emissivity, thermal radiation, temperature, thin films, optical coatings, microcavity

\section{INTRODUCTION}

Optical coatings have been extensively characterized these last decades, due to more and more severe requirements. These characterizations include optical properties, uniformity, hardness, adhesion and stress, damage threshold, absorption, scattering and others... For applications involving mirrors for gyro-laser systems, detection of gravitational waves and space micro-optics, huge efforts were developed to overpass the "ppm barrier", though absorption and scattering still limit the energy balance to a few ppm losses.

Despite this state of the art, thermal radiation [1], [2] in optical coatings was rarely investigated in detail until now, while emissivity [2] plays a key role in numerous sectors related to energy and defense, space optics and MIR imaging... Thermal radiation in multilayers originates from both the ambient and the absorption-induced temperature, and has mostly been studied for opaque samples or for transparent samples supporting a constant temperature in time. Predictions can be found in the literature [2], [3], [4], [5] but often rely on approximations or assumptions, and are rarely connected to photo-induced effects in optical coatings.

The scope of this work is to provide an exact theory of thermal radiation in optical multilayers submitted to an arbitrary illumination (continuous, pulsed, modulated). In a first part, the modelling of the laser induced temperature in optical coatings is discussed and some examples are given for a quarter wave mirror submitted to nanosecond and millisecond pulses. Then the thermal radiation is linked to the temperature thanks to the fluctuation dissipation theorem. Finally, ideas to build planar optical coatings that radiate thermically at specific wavelength and angle are given.

\section{PHOTO-INDUCED TEMPERATURE WITHIN MULTILAYERS OPTICS}

Thermal radiation of optical coatings originates from both the ambient and the photo-induced temperature that occurs when the sample is submitted to an optical illumination. Hence, the first section of this article is dedicated to the modelling of this laser induced temperature.

\subsection{Theory modelling}

Figure 1 shows the geometry of an optical multilayer with $\mathrm{p}$ thin film layers. The media are considered isotropic and homogenous. For each layer (i), the thickness is noted $\mathrm{e}_{\mathrm{i}}$, the refractive index $\mathrm{n}_{\mathrm{i}}$, the thermal conductivity $\mathrm{b}_{\mathrm{i}}$ and the thermal diffusivity $a_{i}$. The superstrate has the refractive index $n_{0}$ whereas the substrate has the index $n_{s}$. The illumination is coming from the superstrate. 


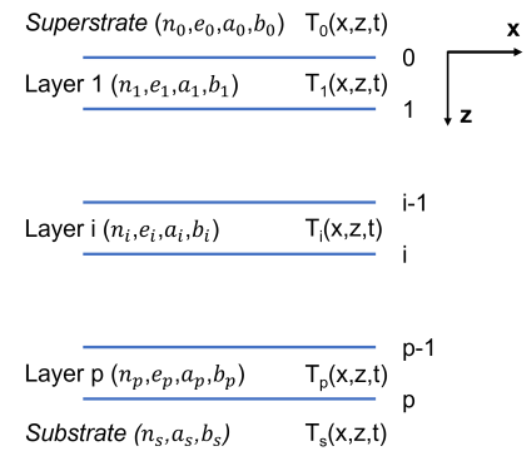

Figure 1: geometry of an optical multilayer (see text)

In this work we focus on the conduction process (heat diffusion), so that heat transfer by convection in the surrounding media is not considered. Hence the temperature in each layer (i) is governed by the classical heat equation:

$$
\Delta T_{i}(\vec{r}, z, t)-\left(\frac{1}{a_{i}}\right) \partial_{t} T_{i}(\vec{r}, z, t)=-\left(\frac{1}{b_{i}}\right) S_{i}(\vec{r}, z, t)
$$

with $T_{i}$ is the photo-induced temperature, $\mathrm{t}$ is the temporal variable, $\vec{r}=(x, y)$ the transversal space coordinates and $\mathrm{z}$ the perpendicular direction to the interfaces of the component. $S_{i}$ is the thermal source and represents the absorption density created by the optical illumination in the media (i).

It is important to notice that this model is acceptable only if the pulse duration is longer than 10 picoseconds. To tackle the temperature induced by ultra-short pulses, one needs to consider the two-temperature model [6].

The resolution of the heat equation has been widely studied in the literature. A lot of papers have considered finite difference algorithms [7], [8], [9], [10], [11] and [12]. Others have used Hankel transform or Laplace transform [13], [14]. In this article, we choose to rely on an analogy between optical propagation and heat diffusion [15] that allows to reuse algorithms that have been developed for the optical propagation in multilayer systems. To use this analogy, one needs to take a double Fourier transform of the heat equation versus the time variable $t$ and the transverse coordinates $\vec{r}$ :

$$
\partial_{z}^{2} \widehat{T}_{i}(\vec{v}, z, f)+\alpha_{i}^{2}(f, v) \widehat{T}_{i}(\vec{v}, z, f)=-\left(\frac{1}{b_{i}}\right) \hat{S}_{i}(\vec{v}, z, f)
$$

where:

$$
\alpha_{i}^{2}(f, v)=j\left(\frac{2 \pi f}{a_{i}}\right)-4 \pi^{2} v^{2} \text { and } v=|\vec{v}|=\sqrt{v_{x}^{2}+v_{y}^{2}}
$$

and the double Fourier transform is written as follow:

$$
\widehat{T}(\vec{v}, z, f)=\iint T(\vec{r}, z, t) e^{-j 2 \pi \vec{v} \cdot \vec{r}} e^{j 2 \pi f t} d^{2} \vec{r} d t
$$

From a mathematical perspective, equation (2) is similar for heat diffusion and optical propagation in absorbing media. The solution of (2) is the sum of the homogenous solution $\widehat{T}_{h, i}$ and a particular solution $\widehat{T}_{g, i}$.

$$
\widehat{T}_{i}(\vec{v}, z, f)=\widehat{T}_{h, i}(\vec{v}, z, f)+\widehat{T}_{g, i}(\vec{v}, z, f)
$$

The homogenous solution is classically given by the following equation:

$$
\widehat{T}_{h, i}(\vec{v}, z, f)=T_{i-1}^{+}(\vec{v}, f) e^{j \alpha_{i}(f, v) z}+T_{i-1}^{-}(\vec{v}, f) e^{-j \alpha_{i}(f, v) z}
$$

The constants $T_{i-1}^{ \pm}$are determined thanks to the continuity of both the temperature and the heat flux at each interface of the component [8].

The particular solution is the convolution product (with respect to the $\mathrm{z}$ variable) between the thermal source and the Green function. 


$$
\widehat{T}_{g, i}(\vec{v}, z, f)=G_{i}(\vec{v}, z, f) *_{z}\left(\frac{-1}{b_{i}}\right) \hat{S}_{i}(\vec{v}, z, f) \text { with } G_{i}(\vec{v}, z, f)=\frac{1}{2 j \alpha_{i}(f, v)} e^{j \alpha_{i}(f, v)|z|}
$$

The last step of the modelling is the determination of the thermal source $S_{i}$ which is the absorption density $\frac{\partial \mathcal{A}_{i}}{\partial v}$ of the incident laser beam in the layer (i). In the most general form, one can write:

$$
\frac{\partial \mathcal{A}_{i}}{\partial v}=E_{i} \partial_{t} D_{i}+H_{i} \partial_{t} B=S_{i}(\vec{r}, z, t) \text { with } D_{i}=\varepsilon_{i} *_{t} E_{i} \text { and } B_{i}=\mu_{i} *_{t} H_{i}
$$

This expression can be greatly simplified by considering two reasonable assumptions. First, the laser is assumed to be quasi monochromatic around a central frequency $\mathrm{f}_{0}$. Then the divergence of the laser beam is assumed to be weak which means that the optical source is collimated. The incident laser beam occurs from one direction described with the spatial frequency $v_{0}=n_{0} \sin \theta_{0} / \lambda_{0}$ where $\theta_{0}$ is the angle of incidence and $\lambda_{0}$ the central wavelength of the laser. With these two assumptions one can write:

$$
S_{i}(\vec{r}, z, t)=\partial_{z} \mathcal{A}_{i}\left(v_{0}, z, \mathrm{f}_{0}\right) g^{2}(\vec{r}, z, t)
$$

with $\mathcal{A}_{i}\left(v_{0}, z, \mathrm{f}_{0}\right)$ the monochromatic and monodirectional absorption and $g^{2}$ a function that describe the shape and the temporal envelope of the laser. The absorption is described by the classical formulation (10) where $\left(\widehat{E}_{0}^{+}, \widehat{H}_{0}^{+}\right)$is the double Fourier transform of the incident electric and magnetic field:

$$
\partial_{z} \mathcal{A}_{i}\left(v_{0}, z, \mathrm{f}_{0}\right)=\pi f_{0}\left\{\operatorname{Im}\left(\epsilon_{i}\right)\left|\frac{\hat{E}_{i}\left(v_{0}, z, f_{0}\right)}{\hat{E}_{0}^{+}\left(v_{0}, f_{0}\right)}\right|^{2}+\operatorname{Im}\left(\mu_{i}\right)\left|\frac{\widehat{H}_{i}\left(v_{0}, z, f_{0}\right)}{\widehat{E}_{0}^{+}\left(v_{0}, f_{0}\right)}\right|^{2}\right\}
$$

To go further, one can assume that the incident beam can be described by separating the temporal variable and the spatial one. If we choose a gaussian laser in time and space, then $g^{2}$ can be written as follow:

$$
g^{2}(\vec{r}, z=0, t)=g_{1}(\vec{r}) g_{2}(t) \text { with } g_{1}(\vec{r})=g_{01} e^{-\frac{2\left(x^{2} \cos ^{2} \theta_{0}+y^{2}\right)}{L^{2}}} \text { and } g_{2}(t)=g_{02} e^{-\frac{2 t^{2}}{\tau^{2}}}
$$

$\mathrm{L}$ represents the width of the beam at $e^{-2}$ and $\tau$ the pulse duration.

Finally, the amplitude of the two gaussian functions can be linked to the energy of the laser pulse $\mathrm{W}$ and the thermal source can be expressed, in the second Fourier plane, as:

$$
\widehat{S}_{i}(\vec{v}, z, f)=\partial_{z} \mathcal{A}_{i}\left(v_{0}, z, \mathrm{f}_{0}\right) \frac{2 W}{\Re\left\{\tilde{n}_{0}\right\}} e^{-\frac{\pi^{2} f^{2} \tau^{2}}{2}} e^{-\frac{\pi^{2} L^{2}}{2}\left(\frac{v_{x}^{2}}{\cos ^{2} \theta_{0}}+v_{y}^{2}\right)}
$$

In the following, the illuminated surface on the top interface of the sample will be large and we will be interested in the depth variation of the temperature field. In these conditions, the incident wave can be assimilated to a plane wave and one can consider a surface energy density. The system becomes invariant in $\mathrm{x}, \mathrm{y}$, and the second Fourier transform is not necessary. Therefore, in the first Fourier plane, the heat equation becomes:

$$
\partial_{z}^{2} \widetilde{T}_{i}(z, f)+\frac{j 2 \pi f}{a_{i}} \widetilde{T}_{i}(z, f)=-\left(\frac{1}{b_{i}}\right) \widetilde{T}_{i}(z, f)
$$

with the thermal source:

$$
\tilde{S}_{i}(z, f)=\partial_{z} \mathcal{A}_{i}\left(v_{0}, z, \mathrm{f}_{0}\right) \frac{2}{R\left\{\tilde{n}_{0}\right\}} \frac{\partial \mathrm{W}}{\partial \mathrm{S}} e^{-\frac{(\pi \tau f)^{2}}{2}}
$$

$\frac{\partial \mathrm{W}}{\partial \mathrm{S}}$ can be seen as the fluence of the illumination.

\subsection{Numerical results}

In this section, the laser-induced temperature of a mirror made of seven quarter wave layers is computed. The high index material is $\mathrm{Nb}_{2} \mathrm{O}_{5}$, and the low index is $\mathrm{SiO}_{2}$. The formula of the component is: $\mathrm{Air} / \mathrm{H}(\mathrm{BH})^{7} / \mathrm{BK} 7$. The optical and thermal properties chosen for the materials are summarized in Table 1. 


\begin{tabular}{|c|c|c|c|c|}
\hline Materials & $\mathrm{Nb}_{2} \mathrm{O}_{5}$ & $\mathrm{SiO}_{2}$ & Air & BK7 \\
\hline $\begin{array}{c}\text { Refractive index } @ \\
1 \mu \mathrm{m}\end{array}$ & $2.25+10^{-4} \mathrm{j}$ & $1.45+10^{-6} \mathrm{j}$ & 1 & 1.52 \\
\hline $\begin{array}{c}\text { Thermal conductivity } \\
(\mathrm{W} / \mathrm{m} / \mathrm{K})\end{array}$ & 1 & 0.5 & $2.5 * 10^{-2}$ & 1.14 \\
\hline $\begin{array}{c}\text { Thermal diffusivity } \\
\left(\mathrm{m}^{2} / \mathrm{s}\right)\end{array}$ & $4.3 * 10^{-7}$ & $1.84 * 10^{-7}$ & $2.05^{*} 10^{-5}$ & $6.2 * 10^{-7}$ \\
\hline
\end{tabular}

Table 1: Optical and thermal parameters used for the simulations of the laser-induced temperature

We first study the laser induced temperature in the nanosecond regime. The laser has an energy of one mJ over one nanosecond. The central wavelength of the laser is of $1064 \mathrm{~nm}$. The illuminated surface is of $100 \mu \mathrm{m}$ by $100 \mu \mathrm{m}$, which gives a fluence of $10^{3} \mathrm{~J} / \mathrm{cm}^{2}$. Note here that laser-induced damage processes are not considered. We are interested in the link between temperature and incident energy. This link is linear and can be used to predict the temperature in other situations. The photo-induced temperature in this configuration is given in Figure 2.

The temperature is also studied in the millisecond regime. The properties of the laser and the materials are the same, except for the pulse duration chosen at one millisecond. Results are given in Figure 3.
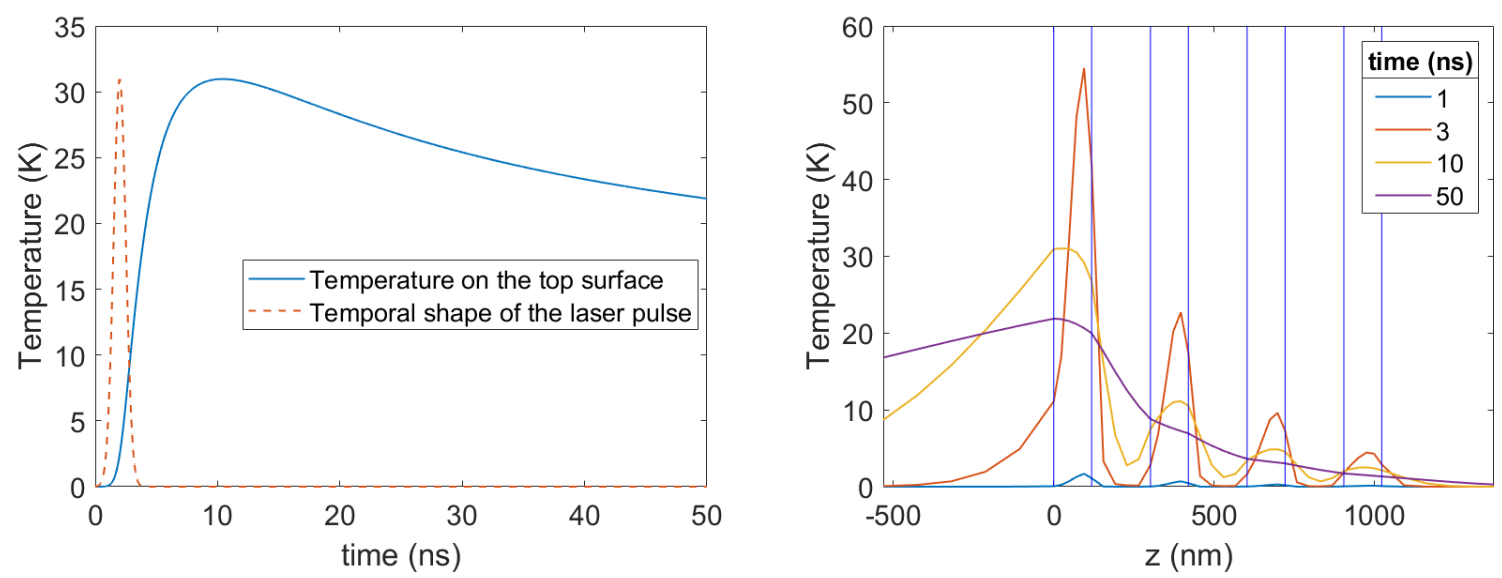

Figure 2: Laser-induced temperature of a quarter wave stack illuminated by a nanosecond pulse (see text). (left) time dependence of the temperature at the top surface of the mirror and of the gaussian shape of the laser pulse. (right) temperature at fixed time with respect to the stack depth $\mathrm{z}$.
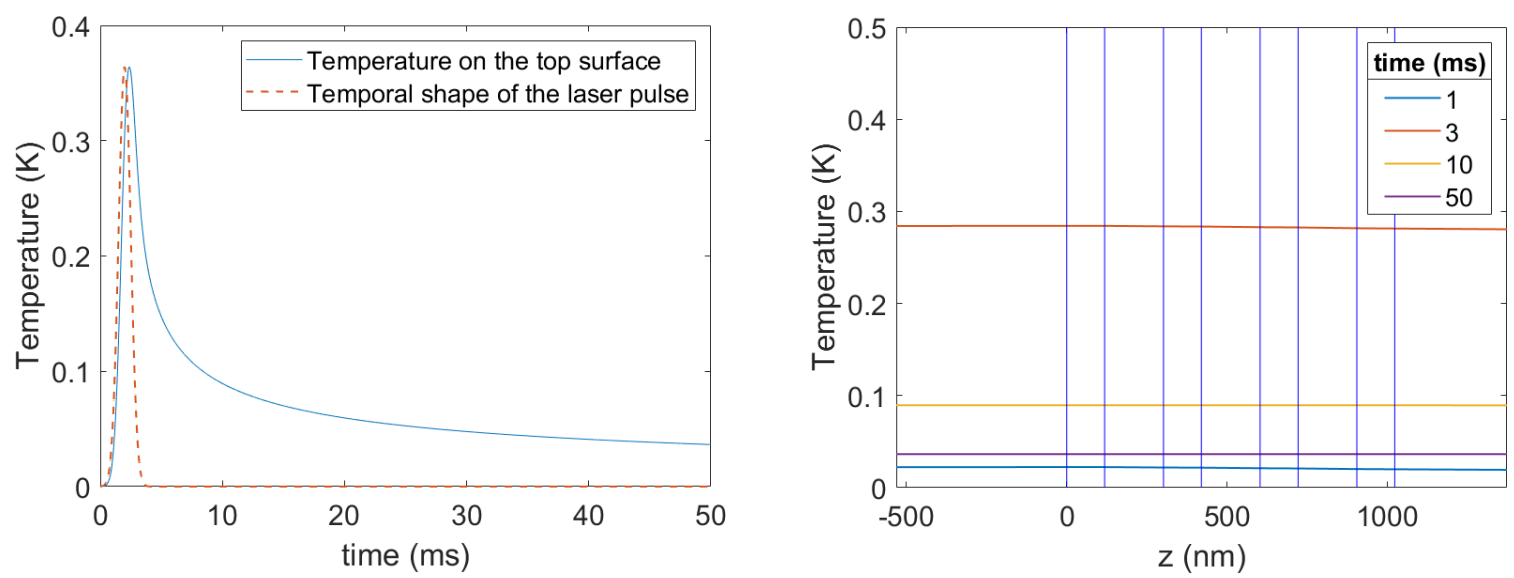

Figure 3: Laser-induced temperature of a quarter wave stack illuminated by a millisecond pulse (see text). (left) time dependence of the temperature at the top surface of the mirror and of the gaussian shape of the laser pulse. (right) temperature at fixed time with respect to the stack depth $\mathrm{z}$.

In the nanosecond regime, the temperature distribution along the direction of depth in the coating follows the normalized modulus squared of the tangential electric field (Figure 4) whereas in the millisecond regime the distribution profile is 
almost monotonic. This can be explained with the thermal diffusion length $L=\sqrt{a \tau}$ which is equal, for the $\mathrm{Nb}_{2} \mathrm{O}_{5}$, to 20.7 $\mathrm{nm}$ in the ns regime, and to $20.7 \mu \mathrm{m}$ in the ms regime. In the ns regime, the temperature field mostly stays in the layers and so follows the thermal source, which is proportional to the electric field. Conversely, in the ms regime the temperature field diffuses along the depth of the thin film which allows the temperature to stabilize.

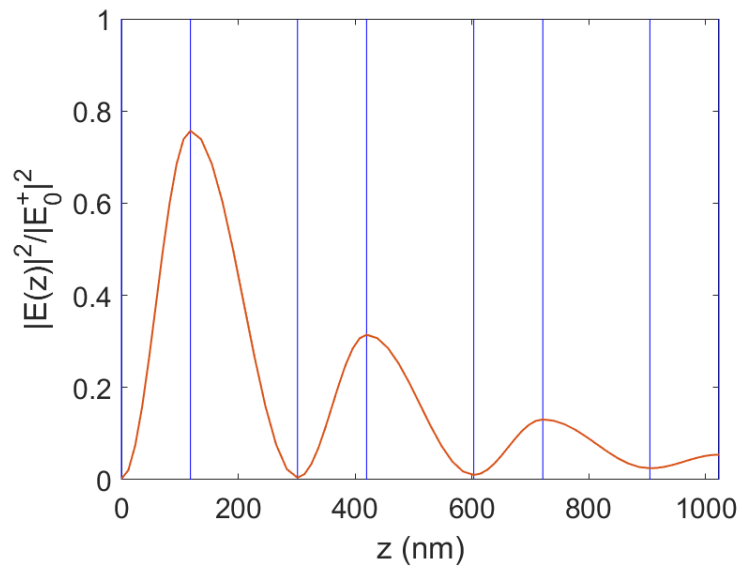

Figure 4: Variation with stack depth $\mathrm{z}$ of the normalized modulus squared of the tangential electric field

Because of the confinement of the temperature field, the ns regime may give the opportunity to solve different inverse problems such as the extraction of the thermal parameters of the different layers from the measurements.

\section{PHOTO-INDUCED THERMAL RADIATION WITHIN MULTILAYER OPTICS}

The aim of this section is to develop the model of the laser-induced thermal radiation of an optical thin film filter submitted to an arbitrary illumination.

\subsection{Theory modelling}

The previous section gives a map of the photo-induced temperature in the coating with respect to the time. This map is discretised in the space and the time domain. The steps of the discretisation are chosen to obtain areas where the temperature is considered as a constant. This discretisation is essential to satisfy the hypothesis of thermal equilibrium that is needed to apply the fluctuation dissipation theorem. Over a given period of time, we place ourselves in the situation of an optical filter where each layer has a different temperature. In this situation, the model of thermal radiation in an 1D multilayer structure can be found in [3], [4], or [5]. The major steps of the modelling are recalled here.

In each medium at thermal equilibrium (where a temperature can be defined), the random thermal movement of the charged particles can be modelled by a spatial time depend electric current density written $\overrightarrow{J_{e}}(\vec{r}, z, t)$ [1], [16]. The time average of this current density is zero. Rytov [1] has applied the fluctuation dissipation theorem and found a relation between the spatial correlation function of the current at two different locations and the temperature of a local isotropic medium:

$$
\left\langle\tilde{J}_{m, e}(\overrightarrow{\mathrm{r}}, z, f) \tilde{J}_{n, e}^{*}\left(\overrightarrow{\mathrm{r}}^{\prime}, z^{\prime}, f\right)\right\rangle=4 \pi \epsilon^{\prime \prime}(f) \Theta(f, T) \delta_{n m} \delta\left(\overrightarrow{\mathrm{r}}-\overrightarrow{\mathrm{r}}^{\prime}\right) \delta\left(z-z^{\prime}\right)
$$

With:

- $\langle$.$\rangle is a statistical average$

- $\quad m, n \in\{x, y, z\}$ are the coordinates of the current density

- $\quad \tilde{J}_{m, e}(\overrightarrow{\mathrm{r}}, z, f)=\int J_{m, e}(\overrightarrow{\mathrm{r}}, z, t) e^{j 2 \pi f t} d t$

- $\quad \epsilon^{\prime \prime}$ is the imaginary part of the dielectric permittivity of the medium

- $\quad \Theta(f, T)=\frac{h f}{e^{\frac{h f}{k T}-1}}$ is the mean energy of Planck's oscillator at the frequency $\mathrm{f}$ and temperature $\mathrm{T}$ in thermal equilibrium

- $\quad \delta_{n m}$ is the Kronecker symbol 


\section{- $\quad \delta$ is the Dirac function}

To compute the thermal radiation of the medium, one needs to introduce this thermal current density in the Maxwell equations. Most of the literature [3], [4], [5], [17] make the use of the Dyadic Green function to solve this problem. We choose to use the results of the theory of bulk scattering [18] that enable to compute the power spectrum of a bulk distribution of current.

In this work we only consider non-magnetic mediums. To tackle the thermal radiation of magnetic component, an analogue relation of (13) that describes the presence of magnetic current must be considered. The imaginary part of the permittivity will be replaced by the imaginary part of the permeability.

Eventually for a component made of $\mathrm{p}$ layers at thermal equilibrium, the time average of the power of the thermal radiation that merges in the superstrate can be written as:

$$
\left\langle\Phi_{0}(t)\right\rangle=\sum_{i=1}^{p} 2 \int_{f=0}^{+\infty} \int_{\vec{v}=-\infty}^{+\infty} \Re\left\{\tilde{n}_{0}\right\} 4 \pi \epsilon_{i}^{\prime \prime}(f) \Theta\left(f, T_{i}\right) F_{i} d^{2} \vec{v} d f
$$

where $\tilde{n}_{0}$ is the effective index of the superstrate and $F_{i}$ is a function that depends on the optical parameters (effective index, complex admittance) and the polarisation of the illumination.

It is useful to extract the common properties of thermal radiation such as intensity and emissivity [2] from equation (14). The intensity is linked to the monochromatic surface flux density of thermal radiation with the following relation.

$$
\frac{\partial \Phi}{\partial S \partial f \partial \Omega}=I(f, \Omega, T) \cos \theta
$$

where $\mathrm{S}$ and $\mathrm{T}$ are respectively the emissive surface and the temperature of the component, $\Omega$ is the solid angle in which the radiation is propagating, $(\theta, \phi)$ are the spherical coordinates and $\mathrm{f}$ is the frequency of the radiation.

By considering equation (14) and (15), one can find for the intensity of thermal radiation of the layer (i) in the superstrate:

$$
I\left(f, \Omega, T_{i}\right)=2 \frac{k_{0}^{2}}{4 \pi^{2}} \Re\left\{\tilde{n}_{0}\right\} 4 \pi \epsilon_{i}^{\prime \prime}(f) \Theta\left(f, T_{i}\right) F_{i}
$$

with $k_{0}$ the wavevector in the superstrate.

It is usual to normalize this intensity by the intensity of the blackbody $I_{b}(f, T)=2 \frac{k_{0}^{2}}{4 \pi^{2}} \Theta(f, T)$ which introduces the emissivity [2]. One can define, for each layer at thermal equilibrium, its emissivity:

$$
\epsilon_{i}(f, \Omega)=\frac{I\left(f, \Omega, T_{i}\right)}{I_{b}(f, T)}
$$

To conclude this section, it is important to introduce the Kirchhoff's law that equalize the emissivity at a given frequency and angle of a given medium, and the absorption by this medium of an incident beam that originates from the same direction at the same frequency. This relation gives an indirect way to compute the thermal radiation by considering the absorption and allows to verify the computational codes [4].

\subsection{Numerical results}

The thermal radiation of the optical component taken in the section 2 is now studied. The ns regime is chosen because of the higher temperature variations. The ambient temperature is chosen at $20^{\circ} \mathrm{C}(293.15 \mathrm{~K})$. The temporal, angular and wavelength pattern of thermal radiation are shown in Figure 5. Refractive index dispersion is not taken into account. The TE polarisation of the radiation is considered. 

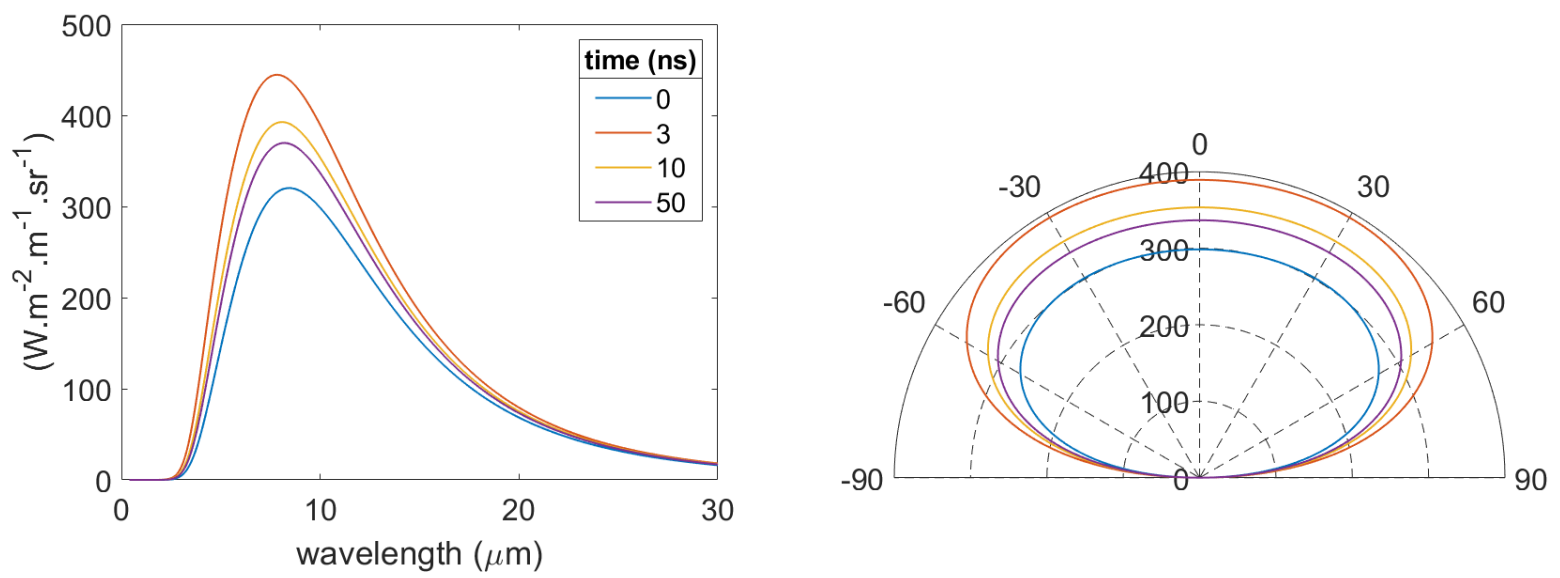

Figure 5: The temporal, angular and wavelength pattern of the thermal radiation of a quarter wave stack illuminated by a nanosecond pulse (see text). (left) wavelength dependence of the intensity at normal incidence and at fixed time. (right) angular dependence at $10 \mu \mathrm{m}$ and at fixed time. Refractive index dispersion is not considered. Patterns in TE polarisation.

In conclusion, the model is accurate to compute the thermal radiation of optical thin film illuminated by an arbitrary laser beam. It should be recall that the model depends on the knowledge of the optical indices of the materials that are poorly known in the infrared region.

\section{TAILORING THE THERMAL RADIATION}

The thermal radiation of solid object at a given temperature is usually quasi-isotropic and spread over a large wavelength bandwidth. Huge efforts have been made to tailor the thermal radiation with the help of nanostructures [5]. Some articles show how to confine the thermal radiation in small wavelength bandwidth or in a small directional aperture thanks to 2D multilayers structures [19] or gratings [20]. Some works are also dedicated to the control of thermal radiation with 1D multilayer structure such as metallic Fabry Perot [4], photonic crystals coated with SiC [21] or Ag [22]. In this article, we demonstrate that we can theoretically achieve coherent thermal radiation with the use of Zero Admittance Layer (ZAL) [23], [24] in 1D dielectric multilayer structures under total internal reflection.

The ZAL layer allows to make a resonant mode of the structure at a specific frequency. At this specific frequency, coherence of thermal radiation can be recovered thanks to the narrow bandwidth. The physical aspects under this phenomenon are similar to those involved in a Fabry Perot filter or in planar micro-microcavities [24],[25],[26].

A multilayer, made of a quarter wave stack with $\mathrm{Nb}_{2} \mathrm{O}_{5}$ and $\mathrm{SiO}_{2}$ for, respectively, the high and low index material, and a ZAL layer of $\mathrm{Nb}_{2} \mathrm{O}_{5}$ has been simulated. The wavelength is chosen at $1 \mu \mathrm{m}$. The structure is assumed to be at thermal equilibrium at a constant temperature of $300 \mathrm{~K}$. Optical parameters of the medium are given in Table 1 . Results are shown in Figure 6. We can see that the thermal radiation is confined in a very narrow angular and wavelength region.

To compare with the literature, the full width at half maximum of the emissivity peak in wavelength $(\Delta \lambda)$ and in angle $(\Delta \theta)$ are computed. We find $\Delta \lambda=0.4 \mathrm{~nm}$, which defined a $\mathrm{Q}$ factor of $Q=\lambda_{c} / \Delta \lambda=2500$, and $\Delta \theta=0.014^{\circ}$ which defines a coherent length of $L_{c}=\lambda / \Delta \theta=71.4 \lambda$. The $\mathrm{Q}$ factor is 10 times higher than those of the structures found in [4], [20], [21] and [22]. The coherence length is also higher. Dahan et al [27] have made a coupled resonant cavity structure that have a larger coherence length but a smaller Q factor. It should be recalled that these results are theoretical ones. The $\mathrm{Q}$ factor and the coherence length will probably be reduced in practice. 

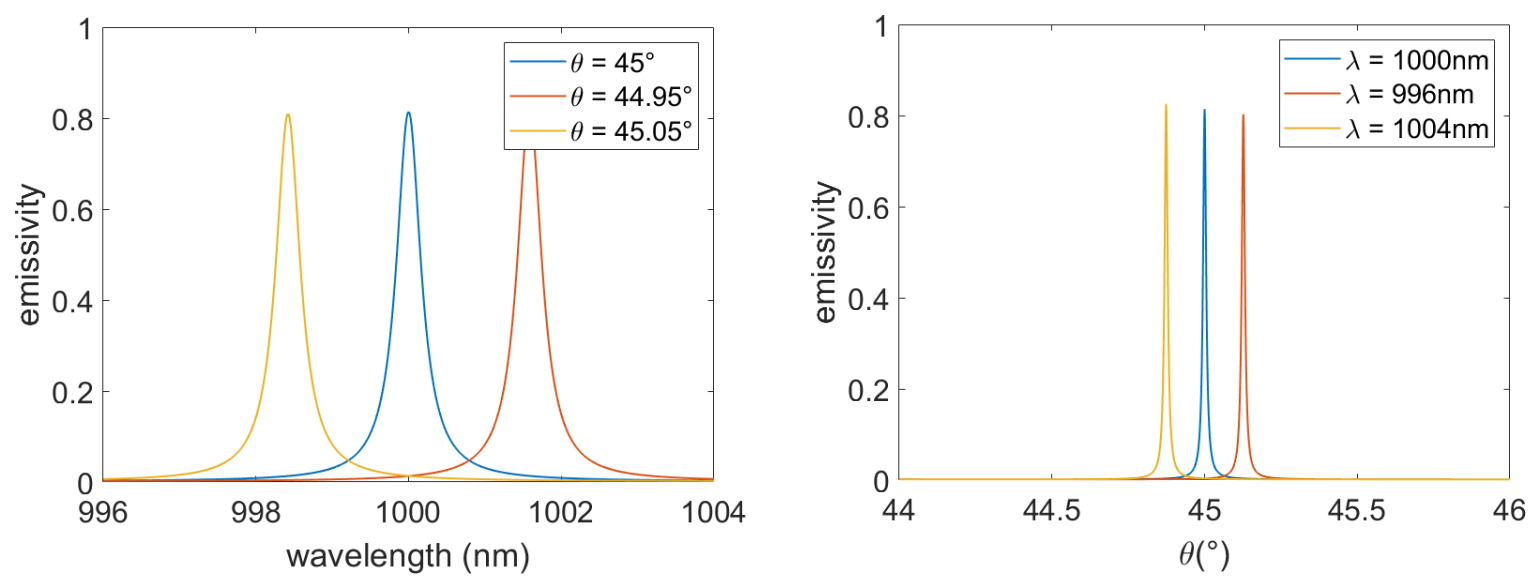

Figure 6: Emissivity of a quarter wave stack with a ZAL layer. (left) wavelength dependence at fixed angles. (right) angular dependence at fixed wavelength

In conclusion, planar multilayer structures made of dielectric are useful to tailor thermal radiation. The $Q$ factor of the structure and the spatial coherence length of the radiation are similar or improved compared to the literature. It is important to highlight the fact that our results were achieved in the near infrared. If one wants to move to the medium infrared region, the materials will have to change to $\mathrm{Ge}, \mathrm{ZnS}$ and so on. Indeed, the optical materials are likely to be opaque in this region preventing the interference effects to occur.

\section{REFERENCES}

[1] S. M. Rytov, Yu. A. Kravtsov, and V. I. Tatarskii, [Principles of Statistical Radiophysics 3: Elements of Random Fields], Springer-Verlag (1989).

[2] John R. Howell, M. Pinar Mengüç, and Robert Siegel, [Thermal Radiation Heat Transfer], $6^{\text {th }}$ edition, CRC Press (2016).

[3] M. Francoeur, M. Pinar Mengüç, and R. Vaillon, "Solution of near-field thermal radiation in one-dimensional layered media using dyadic Green's functions and the scattering matrix method”, Journal of Quantitative Spectroscopy and Radiative Transfer 110 (2009).

[4] L. P. Wang, S. Basu, and Z. M. Zhang, "Direct and Indirect Methods for Calculating Thermal Emission From Layered Structures With Nonuniform Temperatures", Journal of Heat Transfer (2011).

[5] Zhuomin M. Zhang, [Nano/Microscale Heat Transfer], Springer (2020).

[6] Lauren L. Taylor, Jing Xu, Michael Pomerantz, Thomas R. Smith, John C. Lambropoulos, and Jie Qiao, "Femtosecond laser polishing of germanium [Invited]," Opt. Mater. Express 9, 4165-4177 (2019)

[7] J. Crank and P. Nicolson, "A practical method for numerical evaluation of solutions of partial differential equations of the heat-conduction type", Proc. Camb. Phil. Soc. 43 (1947).

[8] Masud Mansuripur, G. A. Neville Connell, and Joseph W. Goodman, "Laser-induced local heating of multilayers", Appl. Opt. 21, 1106-1114 (1982).

[9] Haiyang Hu, Zhengxiu Fan, and Fu Luo, "Laser-induced damage of a 1064-nm ZnS/MgF2 narrow-band interference filter", Appl. Opt. 40, 1950-1956 (2001).

[10] Gang Dai, Yanbei Chen, Jian Lu, Zhonghua Shen, and Xiaowu Ni, "Analysis of laser induced thermal mechanical relationship of HfO2/SiO2 high reflective optical thin film at 1064 nm”, Chin. Opt. Lett. 7, 601-604 (2009).

[11] Bin Wang, Yuan Qin, Xiaowu Ni, Zhonghua Shen, and Jian Lu, "Effect of defects on long-pulse laser-induced damage of two kinds of optical thin films," Appl. Opt. 49, 5537-5544 (2010).

[12] Hongjing Li, Zhonghua Shen and Xiaowu Ni, "Temperature field analysis of high reflection film induced by longpulse and short-pulse lasers under different irradiation angles" Optik 126, 4254-4258 (2015).

[13] M. Lax, "Temperature rise induced by a laser beam", J. Appl. Phys. 48, 3919 (1977).

[14] Agustin Salazar, Arantza Mendioroz, and Alberto Oleaga, "Flying spot thermography: Quantitative assessment of thermal diffusivity and crack width", J. Appl. Phys 127, 131101 (2020). 
[15] C. Amra, D. Petiteau, M. Zerrad, S. Guenneau, G. Soriano, B. Gralak, M. Bellieud, D. Veynante and N. Rolland, "Analogies between optical propagation and heat diffusion: applications to microcavities, gratings and cloaks", Proc. R. Soc. A. 471 (2015).

[16] Lukas Novotny, Bert Hecht, [Principles of Nano-Optics], $2^{\text {nd }}$ edition, Cambridge University Press (2012).

[17] Mulet J-P. Modélisation du Rayonnement Thermique par une Approche Electromagnétique. Rôle des Ondes de Surfaces dans le Transfert d'Energie aux Courtes Echelles et dans les Forces de Casimir. PhD thesis, Université ParisSud 11, Paris (2003) (in French).

[18] C. Amra, "First-order vector theory of bulk scattering in optical multilayers", J. Opt. Soc. Am. A 10, $365-374$ (1993).

[19] Bo Zhao, Liping Wang, Yong Shuai, Zhuomin M. Zhang, "Thermophotovoltaic emitters based on a two-dimensional grating/thin-film nanostructure", International Journal of Heat and Mass Transfer 67, 637-645 (2013).

[20] J.-J. Greffet, R. Carminati, K. Joulain, J.-P. Mulet, S. Mainguy, and Y. Chen, "Coherent emission of light by thermal sources", Nature 416, 61-64 (2002).

[21] B. J. Lee, and Z. M. Zhang, "Coherent thermal emission from modified periodic multilayer structures", Journal of Heat Transfer 129 (2007)

[22] B. J. Lee, Y.-B. Chen, and Z. M. Zhang, "Surface waves between metallic films and truncated photonic crystals observed with reflectance spectroscopy", Optics Letters 33 (2008).

[23] C. Amra et al, "Energy density engineering via zero-admittance domains in all-dielectric stratified materials', Phys. Rev. A. 97, 023819 (2018).

[24] C. Amra, M. Lequime, and M. Zerrad, [Electromagnetic Optics of Thin-Film Coatings: Light Scattering, Giant Field Enhancement, and Planar Microcavities], Cambridge University Press (2021).

[25] Claude Amra and Sophie Maure, "Electromagnetic power provided by sources within multilayer optics: free-space and modal patterns," J. Opt. Soc. Am. A 14, 3102-3113 (1997).

[26] Claude Amra and Sophie Maure, "Mutual coherence and conical pattern of sources optimally excited within multilayer optics," J. Opt. Soc. Am. A 14, 3114-3124 (1997).

[27] Nir Dahan et al, "Extraordinary coherent thermal emission from SiC due to coupled resonant cavities", Journal of Heat Transfer 130 (2008). 